\title{
ALVAC-CEA B7.1 Vaccine
}

National Cancer Institute

\section{Source}

National Cancer Institute. ALVAC-CEA B7.1 Vaccine. NCI Thesaurus. Code C1977.

A cancer vaccine that uses a viral vector system derived from the canarypox virus engineered to target the carcinoembryonic antigen (CEA). It causes infected cells to temporarily display CEA and activates the immune system to attack the tumor cells. 